\title{
Maternal Dietary Intake in Twin Pregnancies: Does it Diminish Towards Term?
}

\author{
Ruth Morley, ${ }^{1,2}$ Mark P. Umstad, ${ }^{3}$ Jodie Bond, ${ }^{2}$ Vivienne M. Moore, ${ }^{4}$ Julie A. Owens, ${ }^{5}$ Terence Dwyer, \\ and John B. Carlin 1,2, \\ ' University of Melbourne Department of Paediatrics, Melbourne, Victoria, Australia \\ ${ }^{2}$ Murdoch Childrens Research Institute, Royal Children's Hospital, Melbourne,Victoria, Australia \\ ${ }^{3}$ Department of Obstetrics, Royal Women's Hospital, Melbourne, Victoria, Australia \\ ${ }^{4}$ University of Adelaide Department of Public Health, South Australia, Australia. \\ ${ }^{5}$ University of Adelaide Department of Obstetrics and Gynaecology, South Australia,Australia
}

W tested the hypothesis that energy intake among women with twin pregnancies decreases in late gestation, as the uterus enlarges and they become increasingly uncomfortable. We asked women to recall dietary intake for the previous 24 hours, every 2 weeks from around 29 weeks of gestation, using a photographic atlas and other strategies to estimate portion size. Eighty women provided data at around 29, 31, 33 and 35 weeks of gestation. We calculated total energy intake, and energy intake from carbohydrate, fat and protein. Data were log transformed to reduce skewness. Using mixed effects linear regression models, we found minimal evidence that total energy intake changed over this time period, either before or after adjustment for potential confounding factors (95\% confidence interval for relative change per week: $-1.4 \%, 0.6 \%)$. There was weak evidence of a small decline in carbohydrate intake over time, both before and after controlling for energy intake. We cannot exclude change in energy intake or diet composition before 29 weeks or after 35 weeks of twin gestation.

There is a dearth of data regarding changes in energy intake in late gestation, but Haste et al. (1990) found that energy intake, measured from 7-day weighed intake records, fell between 28 and 36 weeks among 72 smoking and 89 nonsmoking women $(p<.04$ and $p<.03$ respectively). We hypothesized that energy intake would also decrease over this period in twin pregnancies, as the uterus enlarges and women become increasingly uncomfortable.

We conducted a study of maternal nutrition in twin pregnancies in Melbourne and Adelaide (Morley et al., 2005). We undertook additional data collection in Melbourne to test the primary hypothesis that maternal energy intake decreases beyond 29 weeks of pregnancy and the secondary hypothesis that diet composition changes over this period.

\section{Methods}

This study was approved by the Human Research and Ethics Committees of the Royal Women's Hospital and the Mercy Hospital for Women in Melbourne. Consenting women with twin pregnancies were recruited between 18 and 20 weeks of gestation in the Multiple Pregnancy Clinic at the Royal Women's Hospital, general antenatal clinics at the Mercy Hospital for Women and in the private consulting rooms of two obstetricians at the Royal Women's Hospital. Women were excluded from the study if they were planning to move from the area before delivery, or were unable to understand and complete measures of dietary intake because of insufficient fluency in English.

We used 24-hour recall to assess dietary intake, shown to correlate with mean total energy expenditure in young women (Sawaya et al., 1996). In face-to-face interviews we asked women to recall, 2-weekly from around 29 weeks of gestation, everything they had eaten or drunk over the entire day before their clinic visit. A photographic atlas was used to estimate food portion sizes (Nelson et al., 1997). When there was uncertainty we also asked about portion size in comparison with familiar items (small match-boxes, golf balls, etc.), and sometimes a scale picture of portion size was drawn. We also asked about the brand of all purchased foods or drinks.

Dietary data were analyzed using 'Foodworks' (Xyris software, Queensland, Australia). Weight of, for example, a golf ball-sized portion of potato, or a portion of pizza of specific size was measured.

Received 12 April, 2006; accepted 6 July, 2006.

Address for correspondence: Dr Ruth Morley, University of Melbourne Department of Paediatrics, Royal Children's Hospital, Flemington Road, Parkville, Victoria 3052, Australia. E-mail: morleyr@unimelb.edu.au 
When there was uncertainty about composition of a specific food or drink we referred to recipes or nutritional information for ready-prepared foods. Total energy intake was calculated as the sum of calculated energy intake from carbohydrate, fat and protein.

Mixed effects regression models (incorporating subject-specific slopes and intercepts) were used to assess whether there was a trend to decreased energy intake or change in diet composition, as gestation length increased (Stata Release 9.1, 2005; xtmixed command). Energy values were log transformed to reduce skewness.

\section{Results}

Altogether 172 women were recruited in Melbourne between July 1999 and January 2003. Of these, 28 withdrew from the study, one aborted and another with triplets was recruited in error and subsequently withdrawn by the investigators. Of the 142 remaining in the study, nine were recruited in the pilot phase, before these assessments commenced, and six had delivered or were threatening to deliver by 29 weeks. Therefore 127 women were eligible for this substudy.

All 127 women completed an assessment at around 29 weeks, 123 at around 31 weeks, 108 at around 33 weeks, 80 at around 35 weeks, 32 at around 37 weeks and 5 at around 39 weeks. This attrition was generally because women had delivered, though two women delivering at 37 weeks had only completed the first (29-week) assessment.

For consistency we used data from the 80 women who completed assessments from around 29 weeks until around 35 weeks. As expected, they had a longer mean gestation than the 47 women not included, 37 $(S D=1)$ versus $34(S D=2)$ weeks, but there was little intergroup difference in maternal age $(p=.9)$, prepregnant weight $(p=.4)$, weight at 31 to 33 weeks $(p=$ $.4)$, energy intake at 29 weeks $(p=.7)$, whether first pregnancy $(p=.7)$ and whether monozygotic $(p=.2)$ or monochorionic twins $(p=.4)$.

In Table 1, the median (interquartile range) total energy intake at each time point is shown, and that from each of the macronutrient groups. On average, $52 \%(95 \%$ confidence interval [CI]: $50,54 \%)$ of energy intake was from carbohydrate, $32 \%(95 \%$ CI: $30,33 \%)$ from fat and $16 \%$ (95\% CI: 16, 17\%) from protein.
Dietary intake data were skewed by a few high values at each time-point, so (natural) log values were used in mixed effects regression models to estimate change. There was minimal evidence that total energy intake changed over this time period, either before or after adjustment for maternal age and prepregnant weight (or other potential confounding factors including current weight and maternal smoking). The $95 \%$ CI for change in energy intake per week was $-1.4 \%, 0.6 \%, p=.5$ (Cole, 2000).

There was weak evidence of a decrease in carbohydrate intake over time, both before and after controlling for total energy intake, but little evidence of change in either fat or protein intake. The $95 \%$ confidence intervals for change per week, adjusting for total energy intake, maternal age and prepregnant weight were: for carbohydrate $-1.3 \%, 0.1 \%$, $p=.09$; for fat $-0.3 \%, 1.5 \%, p=.2$; and for protein $-0.6 \%, 1.4 \%, p=.4$.

\section{$\overline{\text { Discussion }}$}

We found little evidence to support the hypothesis that maternal energy intake decreases between 29 and 35 weeks of twin pregnancy. Our results may be interpreted as excluding a mean reduction over the 6-week period of the study of more than about $8 \%$ (since the lower end of the $95 \%$ CI for rate of change in energy intake was $-1.4 \%$ per week, equivalent to a change of $-8.4 \%$ over 6 weeks). Likewise there was little evidence of change in dietary macronutrient composition. However, we cannot exclude change in dietary intake before 29 weeks or after 35 weeks of gestation.

In general, twins are lighter for gestational age than singletons (Hadlock et al., 1991; Min et al., 2000) and this difference increases towards term (Glinianaia et al., 2000; Smith et al., 2001). We considered the possibility that the widening difference in fetal growth between twins and singletons, in the third trimester, is because nutrient intake decreases in late gestation in twin pregnancies by more than in singleton pregnancies. We found no evidence to support this. However, women with twin pregnancies are more likely to be advised regarding optimal weight gain (Luke et al., 2003) than women with singleton pregnancies.

\section{Table 1}

Median (IOR) Total Daily Energy Intake and Energy Intake as Carbohydrate, Fat or Protein in kJ, From Recalled Diet Assessments at 2-Weekly Intervals, in 80 Women With Twin Pregnancies

\begin{tabular}{lcccc}
\hline & At around 29 weeks & At around 31 weeks & At around 33 weeks & At around 35 weeks \\
\hline Median (IQR) total energy intake in kJ & $9902(8161,11,430)$ & $10,006(8182,12,036)$ & $9953(8258,11,029)$ & $9914(7893,11,346)$ \\
Median (IQR) energy intake as carbohydrate in kJ & $5135(4035,5925)$ & $5258(4069,6222)$ & $5195(4051,5969)$ & $4963(3933,5873)$ \\
Median (IQR) energy intake as fat in kJ & $3044(2363,3730)$ & $3172(2453,3957)$ & $3055(2538,3696)$ & $3190(2384,3879)$ \\
Median (IQR) energy intake as protein in kJ & $1565(1254,2023)$ & $1626(1274,1981)$ & $1583(1274,1973)$ & $1540(1224,2029)$ \\
\hline
\end{tabular}

Note: IQR = Interquartile range 


\section{$\overline{\text { Acknowledgments }}$}

This study was funded by the Australian National Health and Medical Research Council (NHMRC). Ruth Morley was supported by the Victorian Health Promotion Foundation (VicHealth) and is now funded by NHMRC. We thank the women who generously participated in this study, and Maggie Flood, who coordinated the study and undertook much of the recruitment and data collection.

\section{$\overline{\text { References }}$}

Cole, T. J. (2000). Sympercents: Symmetric percentage differences on the $100 \log (\mathrm{e})$ scale simplify the presentation of log transformed data. Statistics in Medicine, 19, 3109-3125.

Glinianaia S. V., Skjærven, R., \& Magnus, P. (2000). Birthweight percentiles by gestational age in multiple births. Acta Obstetricia et Gynecologica Scandinavica, 79, 450-458.

Hadlock, F. P., Harrist, R. B., \& Martinez-Poyer, J. (1991). In utero analysis of fetal growth: A sonographic weight standard. Radiology, 181, 129-133.

Haste, F. M., Brooke, O. G., Anderson, H. R., Bland, J. M., Shaw, A., Griffin, J., \& Peacock, J. L. (1990). Nutrient intakes during pregnancy: Observations on the influence of smoking and social class. American Journal of Clinical Nutrition, 51, 29-36.
Luke, B., Hediger, M. L., Nugent, C., Newman, R. B., Mauldin, J. G., Witter, F. R., \& O'Sullivan, M. J. (2003). Body mass index - Specific weight gains associated with optimal birth weights in twin pregnancies. Journal of Reproductive Medicine, 48, 217-224.

Min, S.-J., Luke, B., Gillespie, B., Min, L., Newman, R. B., Mauldin, J. G., Witter, F. R., Salman, F. A., \& O'sullivan, M. J. (2000). Birth weight references for twins. American Journal of Obstetrics and Gynecology, 182, 1250-1257.

Morley, R., Moore, V., Dwyer, T., Owens, J., Umstad, M., \& Carlin, J. B. (2005). The association between erythropoietin in cord blood of twins and size at birth: Does it relate to gestational or perinatal factors? Pediatric Research, 57, 680-684.

Nelson, M., Atkinson, M., \& Meyer, J. (1997) A photographic atlas of food portion sizes. London: MAFF Publications.

Sawaya, A. L., Tucker, K., Tsay, R., Willett, W., Saltzman, E., Dallal, G. E., \& Roberts, S. B. (1996). Evaluation of four methods for determining energy intake in young and older women: Comparison with doubly labeled water measurements of total energy expenditure. American Journal of Clinical Nutrition, 63, 491-499.

Smith, A. P. M., Ong, S., Smith, N. C. S., \& Campbell, D. (2001). A prospective longitudinal study of growth velocity in twin pregnancy. Ultrasound in Obstetrics and Gynecology, 18, 485-487. 